УДК 661.63

DOI: $10.15827 / 0236-235 X .131 .523-529$

\title{
Программа моделирования теплофизических процессов в руднотермических печах при изменении управляющих воздействий
}

\author{
А.Ю. Пучков 1, к.т.н., доиент, putchkov63@mail.ru \\ C.B. Панченко 1, д.m.н., npopeccop, tan_pan@inbox.ru \\ M.B. Черновалова ${ }^{1}$, аспирант, 0208margarita@bk.ru \\ ${ }^{1}$ Смоленский филиал Наиионального исследовательского университета МЭИ, \\ 2. Смоленск, 214013, Россия
}

Разработаны алгоритм и ПО для моделирования теплофизических процессов реакторной зоны руднотермической печи в ответ на изменения управляющих воздействий. В основе алгоритма лежат две математические модели, использующие гидродинамический подход при описании образования шлама в электротермических процессах переработки продуктов пеллетирования обжиговых машин в руднотермических печах. Первая модель описывает теплофизические процессы и отражает стехиометрию компонентов реакции и нестационарные состояния фосфорной руднотермической печи при переработке окомкованных техногенных отходов в приближении сосредоточенных параметров. Вторая модель описывает процесс образования шлама и характеризует унос частиц, являющихся источником шламовых загрязнений фосфора на выходе печи. Модель основана на том, что одной из основных причин образования пыли является унос капель расплава барботирующими газами.

Рассматриваемые печи применяются в многостадийном процессе получения желтого фосфора из отходов апатит-нефелиновых руд, в больших количествах скапливающихся в отвалах горно-обогатительных комбинатов. Являясь техногенными месторождениями минерального сырья, такие отвалы нуждаются во вторичной переработке, так как имеют относительно богатый химический состав, который также надо использовать, и в то же время наносят ощутимый урон окружающей среде и здоровью людей. Поэтому разработка технологических систем по утилизации отходов и создание ПО, моделирующего процессы в таких системах с целью их комплексной оптимизации, представляют собой актуальную исследовательскую задачу.

ПО для моделирования теплофизических процессов реакторной зоны рудновосстановительной печи в дальнейшем будет использовано как составная часть программного комплекса, задачей которого является расчет оптимальных (энергоресурсоэффективных) режимов технологической системы, включающей в себя грануляторы, обжиговые машины конвейерного типа и руднотермические печи, с целью обеспечения экологически безопасной переработки отходов апатит-нефелиновых руд.

Ключевые слова: моделирование теплофизических процессов, оптимизация энергопотребления, утилизация отходов апатит-нефелиновых руд.

Технологические схемы переработки отходов разнообразных производств часто используют энергоемкие агрегаты, для которых относительно небольшая оптимизация режимов работы может дать значительную экономию материальных ресурсов в абсолютном выражении. Пример таких схем - получение желтого фосфора из отходов апатит-нефелиновых руд, хранящихся в отвалах и являющихся результатом производственной деятельности горнообогатительных комбинатов.

Особенно актуальна проблема переработки техногенных отходов для Мурманской области, где сосредоточены крупные перерабатывающие мощности, за счет чего оказывается значительное негативное воздействие на окружа- ющую среду и здоровье населения [1]. Одним из направлений снижения экологического урона от скоплений техногенных отходов может быть их повторное использование для получения минерального сырья, так как химический состав таких отвалов достаточно разнообразен [2]. Учитывая сложность происходящих процессов в химикоэнерготехнологической системе (ХЭТС) производства фосфора, актуальной становится задача разработки математического аппарата для их описания и соответствующего ПО, позволяющего просчитывать режимы функционирования ХЭТС с целью оптимизации $[3,4]$.

Многостадийная технологическая схема получения фосфора из отходов апатит-нефелино- 
вых руд включает несколько последовательно расположенных агрегатов: гранулятор (формирует сырые окатыши из поступающего сырья), многокамерную обжиговую машину конвейерного типа (удаляет влагу из окатышей и обжигает их) и руднотермическую печь (РТП), в которой происходит расплав окатышей с выделением фосфора в газообразной форме.

Программные модели отдельных агрегатов и процессов рассматриваемой ХТЭС уже создавались, например [5, 6], однако для получения комплексной модели, описывающей всю технологическую цепочку получения фосфора, необходимо разработать модели еще ряда подсистем и процессов. Решению одной из таких задач - созданию программной модели для обобщенного описания РТП, в реакционной зоне которой происходят теплофизические процессы, посвящена данная работа.

Теплофизические процессы обеспечивают интенсификацию тепломассопереноса, влияют на экономичность всей РТП, надежность работы ее элементов и показатели качества готового продукта (фосфора). Кроме того, математические модели тепловых и газодинамических процессов могут быть использованы для оптимизации режимов работы технологического оборудования, а также расчетов параметров технологических агрегатов при проектировании [7].

В основе предложенного алгоритма лежат две математические модели, использующие гидродинамический подход при описании образования шлама в электротермических процессах переработки продуктов пеллетирования в РТП. Первой из предложенных моделей является оригинальная одномерная модель теплофизических процессов, отражающая стехиометрию компонентов реакции и нестационарные состояния фосфорной РТП при переработке окомкованных техногенных отходов в приближении сосредоточенных параметров. Модель позволяет имитировать функционирование печи в различных режимных ситуациях и вариациях характеристик перерабатываемого сырья с учетом взаимосвязи реакционной и шихтовой зон, а также описать двойственные режимы работы реактора, формирующиеся при различных размерах коксовой зоны. Вторая модель описывает процесс образования шлама и характеризует унос частиц, являющихся источником шламовых загрязнений фосфора на выходе печи. Модель основана на том, что одной из главных причин образования пыли является унос капель расплава барботирующими газами.
Рассмотрим физико-химические и математические аспекты применяемых моделей. В соответствии с фазовым составом и механизмом физико-химических превращений ванна реактора для восстановления фосфора условно подразделяется на следующие зоны: шихтовая, плавления, углеродистая, шлаковая, ферросплавная [8]. Основой для разработки модели реактора служит реакция восстановления фосфора, которую можно записать в обобщенной форме: $\mathrm{P}_{2} \mathrm{O}_{5}+5 \mathrm{C} \rightarrow 5 \mathrm{CO}+\mathrm{P}_{2}$. Это соотношение позволяет отразить стехиометрию компонентов реакции. Описание нестационарных состояний фосфорной РТП в приближении сосредоточенных параметров дало возможность проводить анализ поведения объекта при различных значениях управляющих параметров, то есть имитировать состояние РТП в различных режимных ситуациях.

Входной вектор модели содержит следующие компоненты: $X=\left(T_{s}, C_{\mathrm{sp} 205}, g_{\mathrm{si}}, g_{\mathrm{ci}}, g_{\mathrm{sli}}, r_{\mathrm{si}}\right.$, $\left.r_{\mathrm{ci}}, r_{\mathrm{sli}}\right)$, где $T_{s}$ - температура шихты на входе в печь; $C_{\mathrm{sp} 205}-$ концентрация пятиокиси фосфора в шихте на входе в печь; $r s_{i}, r c_{i}, r s l_{i}-$ средние радиусы частиц фосфорита, кокса, кварцита соответственно, характеризующие гранулометрический состав шихты (индекс $i$ отражает значения этих величин для различных компонент шихты: $\mathrm{P}_{2} \mathrm{O}_{5}(i=1), \mathrm{CaO}(i=2), \mathrm{MgO}$ $(i=3), \mathrm{SiO}_{2}(i=4), \mathrm{Al}_{2} \mathrm{O}_{3}(i=5), \mathrm{Fe}_{2} \mathrm{O}_{3}(i=6)$, $i=7$ - для других компонентов); $g_{\mathrm{si}}, g_{\mathrm{ci}}, g_{\mathrm{sli}}-$ входные расходы шихты, кокса и кварцита соответственно.

Выходной вектор модели содержит набор параметров: $Y=\left(G_{\mathrm{p}}, C_{\mathrm{lp} 2 \mathrm{o} 5}, j_{\mathrm{el} j}, T_{g}, G_{g}, C_{p g}, G_{l}, T_{l}\right.$, $\left.C_{d g}, M_{k}, \gamma_{\mathrm{p}}\right), j=1, \ldots, 3$, где $G_{\mathrm{p}}$ - расходы фосфора-сырца на выходе из печи; $C_{\mathrm{lp} 205}-$ концентрации пятиокиси фосфора в расплаве; jelj плотность тока $j$-го электрода; $T_{g}$ - температуры газов на выходе в подсводовом пространстве; $G_{g}-$ расход печных газов; $C_{p g}-$ концентрации пятиокиси фосфора в пыли; $G_{l}-$ расход шлака; $T_{l}$ - температура шлака (расплава); $C_{d g}-$ концентрация пятиокиси фосфора в печных газах; $M_{k}-$ модуль кислотности шихты; $\gamma_{p}$ - чистота получаемого фосфора.

Управляющие переменные представлены вектором $U=\left(G_{\mathrm{c}}, G_{\mathrm{sl}}, l_{j}, d y_{j}, U_{f j}, n l\right)$, где $G_{\mathrm{c}}-$ расход кокса; $G_{\mathrm{sl}}-$ расход кварцита; $l_{j}, d y_{j}, U_{j}-$ перемещение, перепуск, фазное напряжение рабочих концов электродов; $n l-$ количество открытых леток. Состояние РТП описывается переменными состояния, собранными в вектор $R=\left(P_{a}, R_{a}, T_{m}, T_{r}, h_{c}, h_{l}, h_{s}, L_{j}, G_{s}\right)$, где $P_{a}-$ активная полезная мощность; $R_{a}-$ сопротивление 
реакционной зоны РТП; $T_{m}, T_{r}$ - температуры плавления шихты и жидкой фазы в реакционной зоне соответственно; $h_{c}, h_{l}, h_{s}$ - высота коксовой зоны, шлака, шихтовой зоны, соответственно; $L_{j}-$ абсолютное положение рабочих концов электродов; $G_{s}-$ расход шихты.

В аналитическом описании программной модели используется аппарат термодинамики, гидравлики. Сложность и недостаточная изученность РТП приводят к необходимости построения математической модели, исследование которой позволит создать предпосылки для разработки эффективной системы управления. Описание нестационарных состояний фосфорной РТП в приближении сосредоточенных параметров для реакционной зоны базируется на уравнениях сохранения массы и энергии [9]. Уравнение энергетического баланса реакционной зоны имеет вид:

$$
\begin{aligned}
& \frac{d}{d \tau}\left(\sum_{i=1}^{3} M_{i} c_{p i} T_{L}\right)= \\
& =\sum_{i=1}^{3} G_{i} c_{p i} T_{m}+P_{a}-G_{g} c_{p g} T_{L}-G_{L} c_{p L} T_{L}-q_{r} w_{r} \gamma_{p} .
\end{aligned}
$$

Сохранение массы компонентов пятиокиси фосфора - основного реагента, расплава, кокса описывается соотношениями:

$$
\begin{aligned}
& \frac{d}{d \tau}\left(M_{L} c_{L \mathrm{P}_{2} \mathrm{O}_{5}}\right)=G_{l} C_{S \mathrm{P}_{2} \mathrm{O}_{5}}-G_{L} C_{L \mathrm{P}_{2} \mathrm{O}_{5}}-w_{r}, \\
& \frac{d}{d \tau}\left(M_{c}\right)=G_{3}-\gamma_{c} w_{r}, \\
& \frac{d}{d \tau}\left(M_{L}\right)=\sum_{i=1}^{3} G_{i}-G_{3}-G_{L}-\left(\gamma_{g}-\gamma_{c}\right) w_{r}
\end{aligned}
$$

с начальными условиями: $\tau=0, M_{L}=\left(M_{L}\right)_{0}$, $T_{l}=\left(T_{l}\right)_{0}, \quad C_{\mathrm{IP}_{2} \mathrm{O}_{5}}=C_{\mathrm{SP}_{2} \mathrm{O}_{5}}, M_{c}=\left(M_{c}\right)_{0}$, где $M_{i}-$ масса $i$-х компонентов (при $i=1$ это параметры $G_{1}$ и $M_{s}$ для фосфорита, при $i=2$ это $G_{2}$ и $M_{L}$ для расплава, при $i=3$ это параметры $G_{3}$ и $M_{c}$ для кокса); $c_{p i}$ - теплоемкость $i$-х компонентов; $w_{r}-$ скорость реакции восстановления $\mathrm{P}_{2} \mathrm{O}_{5} ; q_{r}-$ тепловой эффект реакции; $\gamma_{g}, \gamma_{c}-$ коэффициенты пересчета для печных газов и кокса соответственно; $w_{r}=k_{0} S_{c}\left(1+M_{k}\right)^{m} C_{\mathrm{IP}_{2} \mathrm{O}_{5}} \exp \left(-48000 T_{l}\right)$, $S_{c}=3 M_{c} /\left(\rho_{c} r_{c}\right)-$ поверхность коксовых частиц; $E$ - энергия активации; $k_{0}-$ предэкспоненциальный множитель, $k_{0}=1,6 \times 10^{8}$ кг $\left(\mathrm{M}^{2} \mathrm{c}\right)$.

Расход газов, состоящих в основном из окиси углерода и фосфора, определяется скоростью химических реакций восстановления $G_{g}=\gamma_{g} w_{r}$ и связан условиями стехиометрии: $G_{g}=\frac{5 \mu_{\mathrm{CO}}+\mu_{\mathrm{P}_{2}}}{\mu_{\mathrm{P}_{2} \mathrm{O}_{5}}} w_{r}$, где $\mu_{\mathrm{CO}}, \mu_{\mathrm{P}_{2}}-$ молярные веса оксида углерода и фосфора [10].
Площадь поверхности коксовых частиц, определяемая через их средний радиус $r_{\kappa c p}$ : $S_{c}=3 \frac{M_{c} A_{f}}{\rho_{c} r_{c p}}$, где $M_{c}-$ масса кокса в реакционной зоне; $A_{f}-$ коэффициент формы частиц кокса.

Особенностью работы печи является непрерывная подача шихты в зону реагирования. Такие условия работы обеспечивают саморегулирование объекта, поскольку определяются тепловым состоянием ванны печи [11]. В зависимости от температуры и высоты коксового слоя формируется внутренняя геометрия ванны печи, определяющая состояние равновесия данной системы.

Расход шихты определяется из условий ее плавления на границе жидкой и дисперсной фаз при интенсивном перемешивании расплава за счет барботирования печных газов. Шлак сливается через шлаковые летки периодически или непрерывно под действием гидравлического напора, создаваемого слоем шихты, кокса и шлаковым расплавом. Расход шлака через летки $G_{\mathrm{sl}}=f(\xi) n_{l} S_{L} w_{l}$, где $f(\xi)-$ функция сопротивления при истечении шлака через летки; $S_{L}$ - площадь поперечного сечения летки; $w_{l}-$ скорость шлака на выходе из летки.

Температура шихты и газов в шихтовой зоне определяется из зависимостей для противоточного энергообмена. Модель стационарного состояния печи получается из (1-4).

В алгоритме программной модели учитываются процессы пылеобразования при работе промышленных гетерогенных реакторов (фосфорных, мартеновских и других печей), одной из основных причин которого является унос капель расплава барботирующими газами и их отвердевание наряду с химическими реакциями в газовой фазе с образованием твердых частиц и процессами испарения и конденсации возгонов. На запыленность влияют химический состав шихты, скорость движения газа, электросопротивление расплава, поверхностное натяжение, плотность расплава. Для массы капель в единице массы газа используется зависимость величины уноса капель расплава от скорости газа: $g_{t} \sim c \frac{\rho_{l}}{\rho_{g}} B o_{1}^{0,5}\left(W e^{0,33} B o_{\delta}^{-1}\right)^{n}, \quad$ где $g_{t}$ - масса капель в единице массы газа; $c$ - константа; Bo - критерий Бонда; We - критерий Вебера; $\rho_{g}, \rho_{l}-$ плотность газа и жидкости; $n=2 / s$, где $s-$ дисперсия логарифмически нормального распределения $(s \approx 1) ; B o=\frac{\Delta \rho \times l \times g}{\sigma}$; 
$W e=\frac{\rho_{g} W_{g}^{2} \delta}{\sigma}$, где $l, \delta-$ характерный размер си-

оператора, воспользовавшись опцией «история». В этом случае графически воспроизво- стемы; $\sigma-$ коэффициент поверхностного натяжения жидкой фазы; $g$ - ускорение свободного падения; $\Delta \rho=\rho_{l}-\rho_{g}$.

Схема укрупненного алгоритма разработанного ПО, использующего представленные аналитические зависимости, показана на рисунке 1. Критерием оптимальности управления выступает чистота получаемого фосфора $\gamma_{p}$, являющегося выходным параметром ХЭТС.

Представленная программа дает возможность имитировать режимные задачи при эксплуатации РТП, адаптировать эксплуатационный персонал к экстремальным ситуациям и проводить тренинг по ручному управлению сложным объектом при различных управляющих воздействиях. Средой реализации программы была выбрана Visual Studio 2019 Community $\mathrm{C}++$. Вид главной панели приведен на рисунке 2. На панели управления отображается внутренняя картина зон шихты (вверху), коксовой зоны и зоны расплава (внизу). Показана открытая летка, из которой осуществляется слив шлака (изображен струей). Если открыты две летки, то будут изображаться две струи. Высота зон меняется по ходу процесса, и наблюдателю видны расходование кокса и накопление или убыль уровня шлака. Интерфейс позволяет оператору визуально фиксировать ситуацию внутри объекта управления и находить пути выбора режима, отслеживая поведение основных показателей.

Такая возможность делает обучение оператора более осознанным. При окончании вывода печи на режим можно воспроизвести всю последовательность принимаемых действий

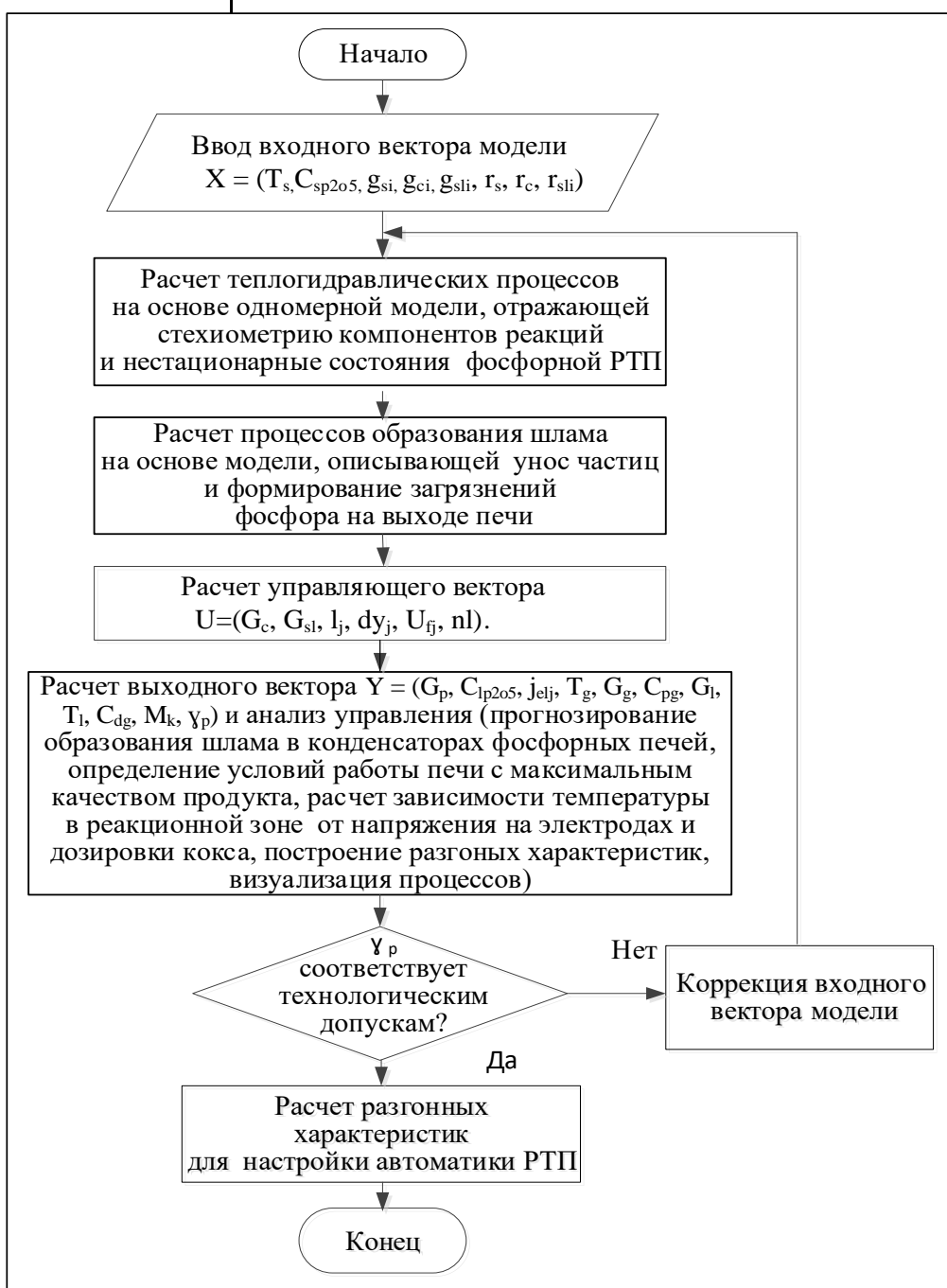

Рис. 1. Схема алгоритма

Fig. 1. Algorithm scheme

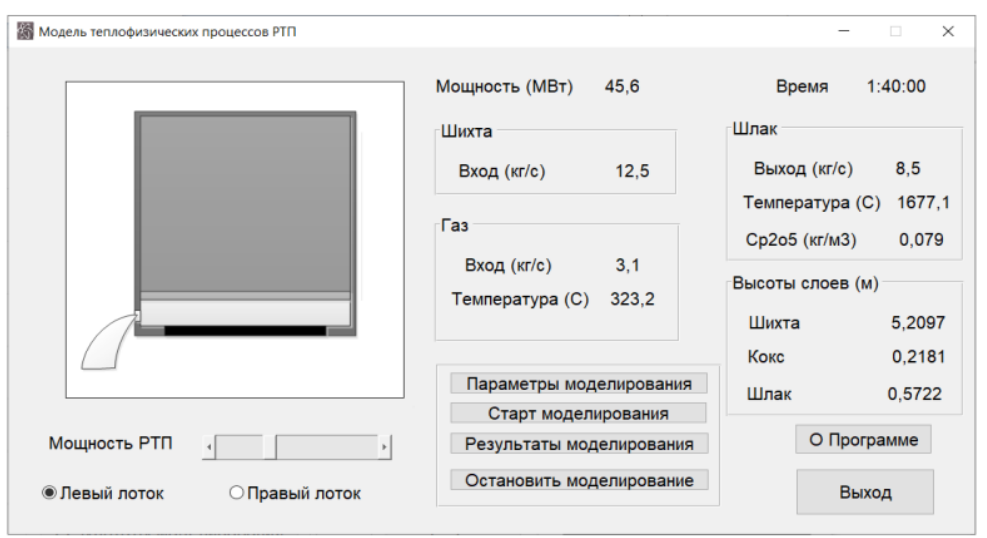

Рис. 2. Главное окно программьл

Fig. 2. The main window of the program 
дится весь процесс выхода печи на режим. Использование концепции виртуальной реальности позволяет повысить оперативность обучения и делает возможным полное понимание процессов, протекающих в печи при управлении. Для стационарных условий работы подобная модель служит базисом для анализа влияния управляющих параметров на основные показатели печи.

Управляющие элементы интерфейса имеют следующее назначение: «Мощность РТП» задает уровень мощности РТП из диапазона [20; 80] МВт; «Параметры моделирования» задает характеристики сырья, оборудования, шаг временной дискретизации и начальные условия для расчетов; «Результаты моделирования» открывает дополнительное окно, в котором можно осуществить выбор параметров для отображения на графиках, а также сформировать отчет в виде текстового файла. Выход на стационарный режим для такого теплоемкого объекта, как печь, занимает много часов. Имея информацию об откликах системы на возмущающие воздействия, программа строит разгонные характеристики объекта управления для настройки автоматики печи. Состояние восстановительной зоны определяет режим работы реактора в целом - там выделяется основное количество тепла и происходит образование продуктов реакции при восстановлении исходных веществ. Управление режимом работы РТП осуществляется изменениями дозировки кокса и напряжения на электродах, поэтому модель позволяет проводить анализ зависимости температуры в реакционной зоне от этих параметров. На их основе определяются концентрация продукта реакции на выходе из реактора, удельный расход электроэнергии и другие технологические параметры.

Программа позволяет получать графики переходных процессов - зависимостей расчетных высот зон печи, температуры шлака и отходящих газов от времени после ступенчатого изменения указанных управляющих воздействий. На рисунке 3 показан пример переходного процесса для расчетных высот зон печи. Используя получаемые зависимости, можно прогнозировать образование шлама в конденсаторах фосфорных печей и определять оптимальные условия работы РТП. Это дает возможность контролировать появление шлама и проводить режимные мероприятия, создавать карты режимов для учета снижения качества продукта при интенсивном образовании пыли. Изменения расчетных размеров зон отображаются на главной форме программы в реальном масштабе времени и позволяют применять программу как тренажер для эксплуатационного персонала.

Проведенные исследования позволили получить математическое описание теплофизических процессов в реакционной зоне РТП и на их основе разработать ПО для анализа этих процессов. Результаты работы могут быть использованы при обучении эксплуатационного персонала, а также при выполнении исследований по созданию методов и алгоритмов комплексного управления сложной ХЭТС по производству фосфора для оценки влияния управляющих воздействий и характеристик сырья на энергоресурсоэффективность переработки техногенных отходов.

Исследование выполнено при финансовой поддержке РФФИ в рамках научного проекта № 18-29-24094. 


\title{
Jumepamypa
}

1. Карначев И.П., Головин К.А., Панарин В.М. Вредные производственные факторы в технологии добычи и переработки апатит-нефелиновых руд Кольского Заполярья // Изв. Тульского гос. ун-та. Естественные науки. 2012. № 1-2. С. 95-100.

2. Архипов А.В., Решетняк С.П. Техногенные месторождения: разработка и формирование. Апатиты: Изд-во КНЦ РАН, 2017. 175 c.

3. Бобков В.И., Борисов В.В., Дли М.И., Мешалкин В.П. Многокритериальная оптимизация энергоэффективности технологических процессов термической подготовки сырья // Теоретические основы химической технологии. 2015. Т. 49. № 6. С. 842-846. DOI: 10.7868/S0040357115060020.

4. Петрышев А.Ю., Берсенев И.С., Боковиков Б.А., Ярошенко Ю.Г. Исследование особенностей формирования оксидов азота при агломерации железных руд // Изв. вузов. Черная металлургия. 2016. T. 59. № 4. С. 232-236. DOI: 10.17073/0368-0797-2016-4-232-236.

5. Meshalkin V.P., Bobkov V.I., Dli M.I., Khodchenko S.M. Computer modeling of the chemical-power engineering process of roasting of a moving multilayer mass of phosphorite pellets. Doklady Chemistry, 2017, no. 477 , vol. 2 , pp. $282-285$. DOI: $10.1134 /$ S0012500817120023.

6. Панченко С.В., Мешалкин В.П., Дли М.И., Борисов В.В. Компьютерно-визуальная модель теплофизических процессов в электротермическом реакторе // Цветные металлы. 2015. № 4. С. 55-60. DOI: $10.17580 /$ tsm.2015.04.10.

7. Meshalkin V.P., Panchenko S.V., Dli M.I., et al. Analysis of the thermophysical processes and operating modes of electrothermic reactor using a computer model. Theor. Found. Chem. Eng., 2018, vol. 52, iss. 2, pp. 166-174. DOI: $10.1134 /$ S0040579518020124.

8. Konevskii M.R. On the kinetic characteristics of the reduction and sublimation of phosphorus from melts. Russian Metallurgy (Metally), 2017, vol. 7, pp. 617-621. DOI: 10.1134/S0036029517070072.

9. Чумаков Ю.А., Мазманян В.А., Румянцев Д.В., Цемехман Л.Ш., Егоров П.А. Анализ работы рудно-термических печей комбината «Печенганикель» в условиях изменения состава шихты // Цветные металлы. 2014. № 1. С. 35-40.

10. Панченко С.В., Широких Т.В. Теплофизические процессы в шихтовой зоне электротермических рудовосстановительных реакторов // Теоретические основы химической технологии. 2014. Т. 48. № 1 . C. 77-81. DOI: $10.7868 / \mathrm{S} 0040357114010096$.

11. Pedro A.A. Thermal monitoring of the electrode and bath in an ore-roasting furnace during downtime. Steel in Translation, 2013, no. 43, pp. 110-112. DOI: 10.3103/S0967091213020149.

\section{The simulation program for thermophysical processes in ore-thermal furnaces when changing the control actions}

\author{
A.Yu. Puchkov ${ }^{1}$, Ph.D. (Engineering), Assistant Professor, putchkov63@mail.ru \\ S.V.Panchenko ${ }^{1}$,Dr.Sc. (Engineering), Professor,tan_pan@inbox.ru \\ M.V.Chernovalova ${ }^{1}$, Postgraduate Student,0208margarita@bk.ru
}

${ }^{1}$ Smolensk Branch of the Moscow Power Engineering Institute, Smolensk, 214013, Russian Federation

\begin{abstract}
The authors developed an algorithm and software for modeling the thermophysical processes of the reactor zone of an ore-thermal furnace in response to changes in control actions. The algorithm is based on two mathematical models that use a hydrodynamic approach to describe the sludge formation in electrothermal processes of the processing of pelleting products of roast machines in ore-thermal furnaces. The first model describes thermophysical processes and represents the stoichiometry of the response components and the nonsteady states of a phosphoric ore-thermal furnace during the processing of pelletized industrial waste in the approximation of lumped parameters. The second model describes the sludge formation process and characterizes the particle entrainment. These particles are a source of sludge contaminants of phosphorus at the furnace outlet. The model is based on the fact that the dusting key factor is the melt drip entrainment by bubbling gas.

The considered furnaces are used in a multistage process for producing yellow phosphorus from the waste of apatite-nepheline ores, which gather in large quantities in the dumps of mining-and-processing integrated
\end{abstract}


works. Representing industrial deposits of mineral wealth, such dumps need to be recycled, since, in addition to the relatively rich chemical composition, which also needs to be used, they have an appreciable loss to the environment and human health. Therefore, the technological system development for waste disposal and the software creation that simulates the processes in such systems with the aim of their comprehensive optimization is a relevant research problem.

The software for modeling the thermophysical processes of the reactor zone of the ore reduction furnace will be further used as an integral part of the software complex, whose problem is to calculate the optimal (energy-efficient) modes of the technological system, including granulators, conveyor-type calciners, and orethermal furnaces in order to ensure environmentally friendly processing apatite-nepheline ore waste.

Keywords: modeling of thermophysical processes, optimization of energy consumption, waste disposal of apatite-nepheline ores.

Acknowledgements. The reported study was funded by RFBR according to the research project no. 18-29-24094.

\section{References}

1. Karnachev I.P., Golovin K.A., Panarin V.M. Harmful production factors in the technology of extraction and processing of apatite-nepheline ores of the Kola Arctic. News of Tula State Univ. Natural Sci., 2012, no. 1-2, pp. 95-100 (in Russ.).

2. Arkhipov A.V., Reshetnyak C.P. Technogenic Deposits. Development and Formation. Apatity: KSC RAS Publ., 2017, 175 p. (in Russ.).

3. Bobkov V.I., Borisov V.V., Dli M.I., Meshalkin V.P. Multicriterial optimization of the energy efficiency of the thermal preparation of raw materials. Theoretical Foundations of Chem. Eng., 2015, vol. 49, no. 6, pp. 842-846. DOI: $10.1134 / \mathrm{S} 0040579515060020$.

4. Petryshev A.Yu., Bersenev I.S., Bokovikov B.A., Yaroshenko Yu.G. Investigation of the features of the formation of nitrogen oxides during agglomeration of iron ores. News of Universities. Ferrous Metallurgy, 2016, vol. 59, no. 4, pp. 232-236 (in Russ.). DOI: 10.17073/0368-0797-2016-4-232-236.

5. Meshalkin V.P., Bobkov V.I., Dli M.I., Khodchenko S.M. Computer modeling of the chemical-power engineering process of roasting of a moving multilayer mass of phosphorite pellets. Doklady Chemistry, 2017, no. 477, vol. 2, pp. 282-285. DOI: 10.1134/S0012500817120023.

6. Panchenko S.V., Meshalkin V.P., Dli M.I., Borisov V.V. Computer-visual model of thermophysical processes in electrothermal reactor. Tsvetnye Metally, 2015, no. 4, pp. 55-60 (in Russ.). DOI: 10.17580/tsm. 2015. 04.10 .

7. Meshalkin V.P., Panchenko S.V., Dli M.I., et al. Analysis of the thermophysical processes and operating modes of electrothermic reactor using a computer model. Theor. Found. Chem. Eng., 2018, vol. 52, iss. 2, pp. 166-174. DOI: 10.1134/S0040579518020124.

8. Konevskii M.R. On the kinetic characteristics of the reduction and sublimation of phosphorus from melts. Russian Metallurgy (Metally), 2017, vol. 7, pp. 617-621. DOI: 10.1134/S0036029517070072.

9. Chumakov Yu.A., Mazmanyan V.A., Rumyantsev D.V., Tsemekhman L.Sh., Egorov P.A. Analysis of orethermal furnace operation at "Pechenganickel" combine under conditions of changing of charge composition. Tsvetnye Metally, 2014, no. 1, pp. 35-40 (in Russ.).

10. Panchenko S.V., Shirokikh T.V. Thermophysical processes in the burden zone of submerged arc furnaces. Theor. Found. Chem. Eng., 2014, vol. 48, no. 1, pp. 77-81 (in Russ.). DOI: 10.1134/S0040579514010096.

11. Pedro A.A. Thermal monitoring of the electrode and bath in an ore-roasting furnace during downtime. Steel in Translation, 2013, no. 43, pp. 110-112. DOI: 10.3103/S0967091213020149.

\section{Для цитирования}

Пучков А.Ю., Панченко С.В., Черновалова М.В. Программа моделирования теплофизических процессов в руднотермических печах при изменении управляющих воздействий // Программные продукты и системы. 2020. Т. 33. № 3. C. 523-529. DOI: 10.15827/0236-235X.131.523-529.

\section{For citation}

Puchkov A.Yu., Panchenko S.V., Chernovalova M.V. The simulation program for thermophysical processes in ore-thermal furnaces when changing the control actions. Software \& Systems, 2020, vol. 33, no. 3, pp. 523-529 (in Russ.). DOI: 10.15827/0236-235X.131.523-529. 